\title{
Desmoid tumor in a laparotomy scar in a child: Case report and brief literature review
}

\author{
J. Skondras ${ }^{1}$, P. Valioulis ${ }^{2}$, D. Papakonstantinou ${ }^{3}$, N. Economopoulos ${ }^{4}$, A. Sourla ${ }^{5}$, N. Zavras ${ }^{3}$ \\ ${ }^{1} 2^{\text {nd }}$ Pediatric Surgery Department, “P\&A Kyriakou” Children’s Hospital, Athens, Greece \\ ${ }^{2}$ Pediatric Surgery Department, Athens Medical Center, Athens, Greece \\ ${ }^{3} 3^{\text {rd }}$ Department of General Surgery, ATTIKO University Hospital, Athens, Greece \\ ${ }^{4} 2^{\text {nd }}$ Department of Radiology, ATTIKO University Hospital, Athens, Greece \\ ${ }^{5}$ Department of Pathology, Athens Medical Center, Athens, Greece \\ Email:nikos4553@gmail.com
}

Received 11 November 2013; revised 1 December 2013; accepted 8 December 2013

Copyright (C) 2013 J. Skondras et al. This is an open access article distributed under the Creative Commons Attribution License, which permits unrestricted use, distribution, and reproduction in any medium, provided the original work is properly cited.

\begin{abstract}
Desmoid tumors are rare neoplasms arising at the site of any fascia. Although histologically benign, they can cause local infiltration and they also carry a risk of recurrence. We describe the case of an 11-year-old girl who presented with a desmoid tumor at the site of an old trauma, and we briefly review the literature of this lesion.
\end{abstract}

Keywords: Desmoid Tumor; Laparaotomy Scar; Children

\section{INTRODUCTION}

Desmoid tumors (DTs), also referred as aggressive fibromatosis (AF), are rare neoplasms arising from fas$\mathrm{cial} /$ muscloaponeurotic stromal structures or soft tissue $[1,2]$. Although histologically benign and with no known potential for metastasis, DTs may cause local infiltration and compression of the surrounding tissues [3]. Furthermore, a high risk of recurrence has been reported despite appropriate surgical treatment [4]. The tumor originates from progenitor mesenchymal myofibroblasts [1]. A number of factors have been shown to be implicated in its pathogenesis, such as genetic mutations most commonly seen in familiar adenomatous polyposis (FAP) and Gardner's syndrome [5], high estrogen levels [6], and trauma-surgical trauma in particular [7]. Herein, we describe the case of a young female patient with an abdominal wall DT located at the site of a previous appendectomy, and briefly discuss the management of such lesions.

\section{CASE REPORT}

An 11-year-old girl presented to the pediatric Emergency
Department, complaining of lower right quadrant abdominal pain. The patient's history was remarkable only for an uncomplicated open appendectomy performed 20 months earlier, with no other history of disease or surgery. She had no relevant family history of FAP or similar conditions. Physical examination revealed a firm, painful mass at the site of the scar of the previous appendectomy. Blood work-up results were normal. A Doppler ultrasound (US) examination revealed a dense fusiform mass, located $1-2 \mathrm{~cm}$ below the old surgical scar in the right lower abdominal wall, measuring $3.31 \times$ $1.62 \times 3.34 \mathrm{~cm}$ with poor internal vascularization. A T1-weighted magnetic resonance imaging study (MRI) (Figures 1(a) and (b)) revealed an enhancing lesion located on the transversalis fascia and aponeurosis of the abdominal muscles on the right. At surgery, the tumor was found to have infiltrated the surrounding muscles; the rectus sheath and the peritoneum were intact. The tumor was radically excised along with part of the internal and external oblique muscles and transversalis fascia. Macroscopic examination identified a firm, oval-shaped, yellow-brown tumor with irregular margins (Figure 2).

Histologic investigation of the specimen revealed a fibroblastic neoplasm consisting of spindle-shaped cells of uniform appearance, set in a collagenous stroma (Figure 3(a)). Immunohistochemistry disclosed Actin positivity (Figure 3(b)), focal staining for Desmin (Figure 3(c)), and low mitotic activity (Ki67) (<2\%) (Figure 3(d)).

The postoperative course was uneventful, and the patient was discharged in good health.

\section{DISCUSSION}

First described in 1832, this lesion acquired its name in 1838 [8]. The term desmoid is of Greek origin and de- 


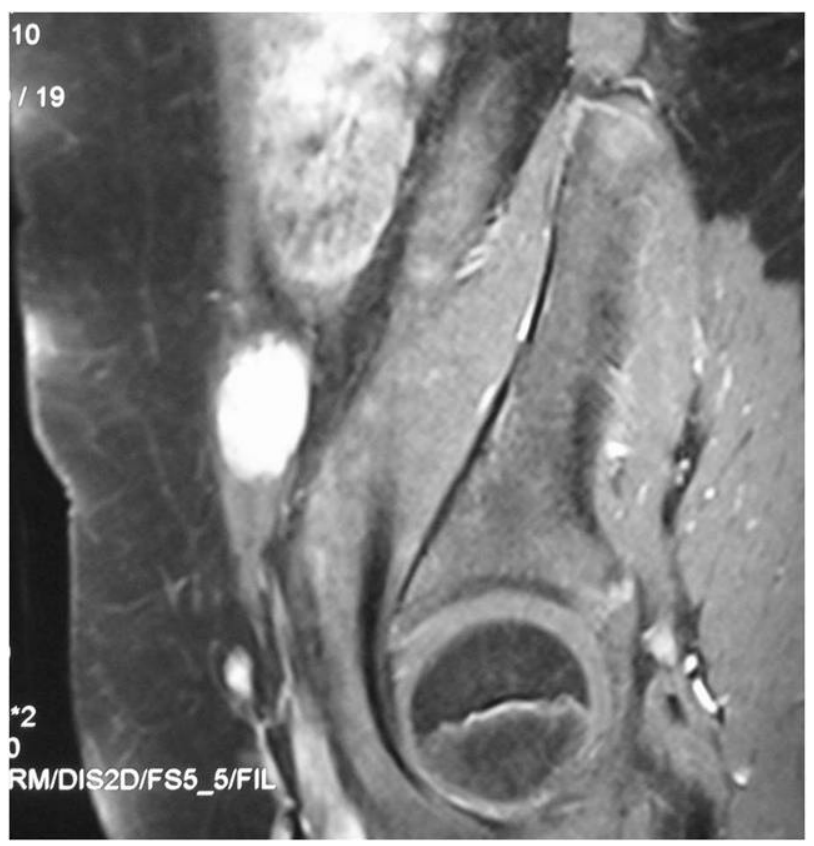

(a)

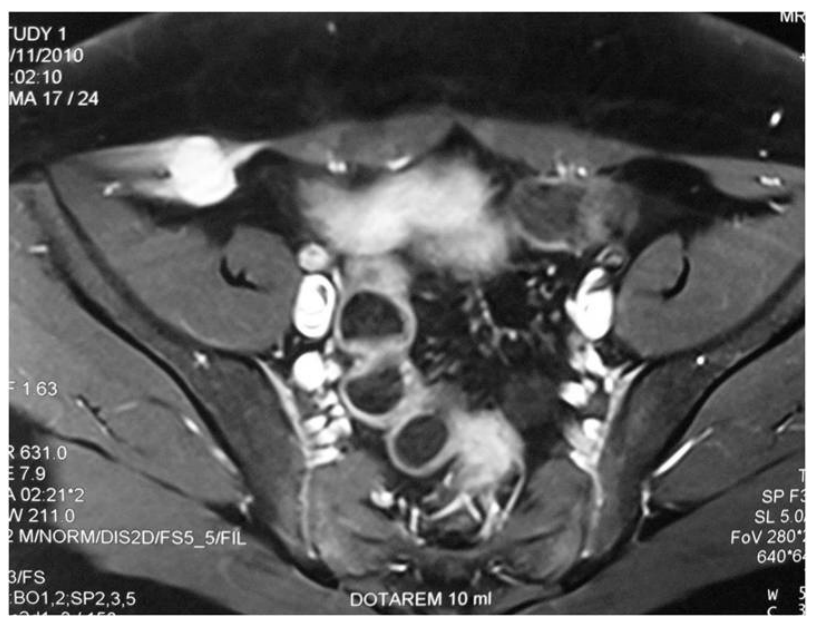

(b)

Figure 1. Transverse (a) and oblique sagittal (b), fat-saturated T1-weighted image of the lower abdomen displays an intensely enhanced lesion measuring 3.31 in diameter $\mathrm{cm}$ and located on the transversalis fascia and aponeurosis of the abdominal muscles, on the right.

rives from the word desmos that means band or tendon [9]. Despite having gained further insight into the nature of the disease, we are still uncertain as to the exact etiology [6]. Its management also remains controversial owing to the unpredictable clinical course of the tumor [10].

The incidence of sporadic cases of DTs observed in the general population is estimated to be 2 to 4 new cases per million people per year, and represents about $0.03 \%$ of all neoplasms and $3 \%$ of all soft tumors [2]. In FAP patients, it is approximately 850 times than that of the general population [11]. Among the pediatric population,

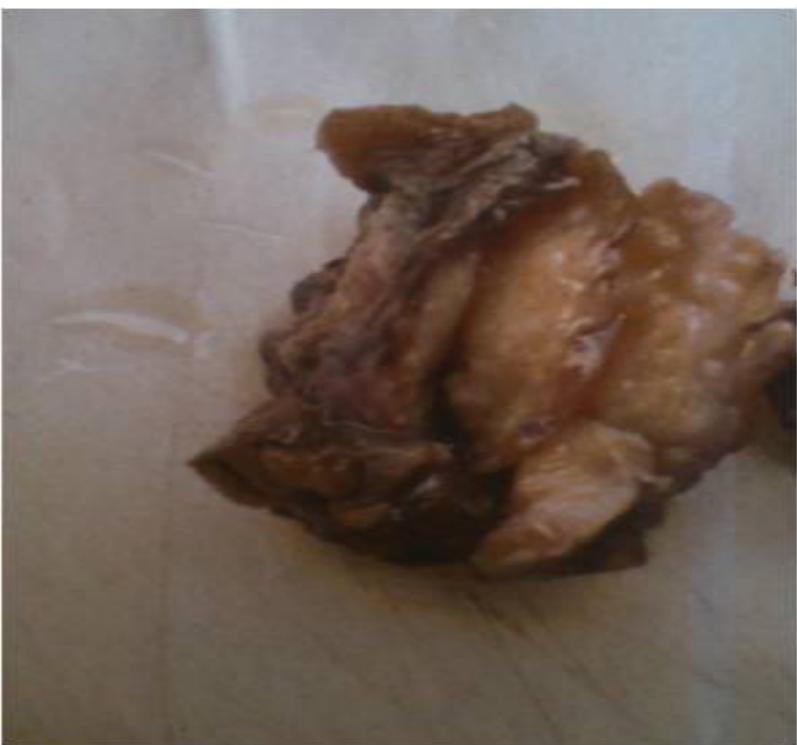

Figure 2. Surgical specimen showing a firm, yellow-brown mass with abnormal margins.

DTs account for less than $1 \%$ of all tumors [10], with peak incidence at 4.5 years (range 0 - 12 years), a female preponderance of $3.3: 1$, and a mainly extra-abdominal distribution [2,11].

Though the exact etiology of DTs remains obscure, an association has been well described with dysregulation of the beta-catenin pathway caused by mutation of the adenomatous polyposis coli (APC) suppressor tumor gene which regulates the cellular levels of beta-catenin [5]. Inherited APC mutation has been found to reach 25\% in FAP patients [12]. In contrast, somatic mutations are rare [13] in sporadic DTs; the latter show a predisposition for direct beta-catenin mutations [14].

Surgical trauma represents one of the factors that possibly contribute to the pathogenesis of sporadic abdominal wall DTs. The tumor usually arises from the musculoaponeurotic structures, especially the rectus and internal oblique muscles and their fasciae [15], and less commonly from the external oblique muscle and transversalis muscle or fascia [16]. Brasfield et al. [17] suggested that the repeated stretch of abdominal muscles, especially the rectus abdominis muscle in women during pregnancy, may result in injury of the epimysium, and lead to the development of a DT. Similarly, as observed in the present case, the stretch of the muscles during separation of the internal oblique and transversus abdominis muscles might cause also injury to the epimysium, and subsequent formation of a DT. The lesion typically presents as a firm, painless nodular mass or abdominal wall swelling, confined to the muscles and the overlying aponeuroses, with the overlying skin intact [18]. Pain manifests with the infiltration of adjacent nerves and muscles, as in our case [11]. 


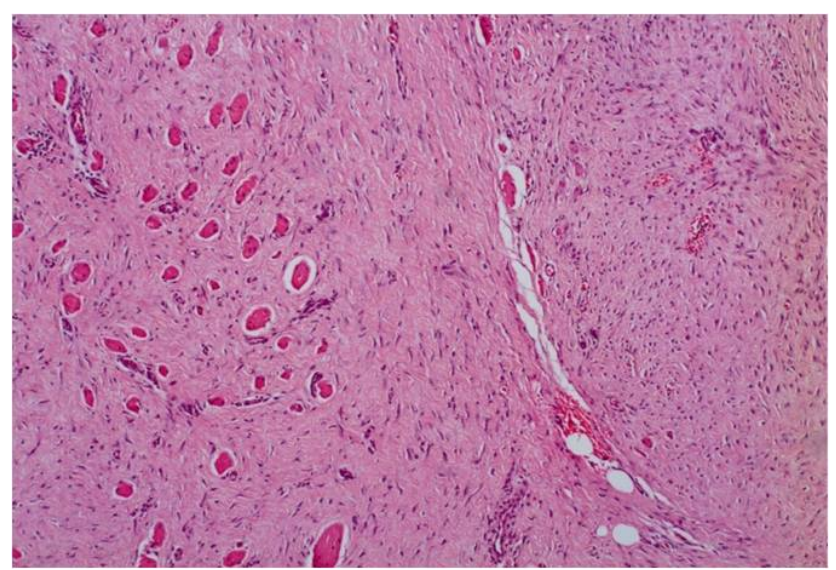

(a)

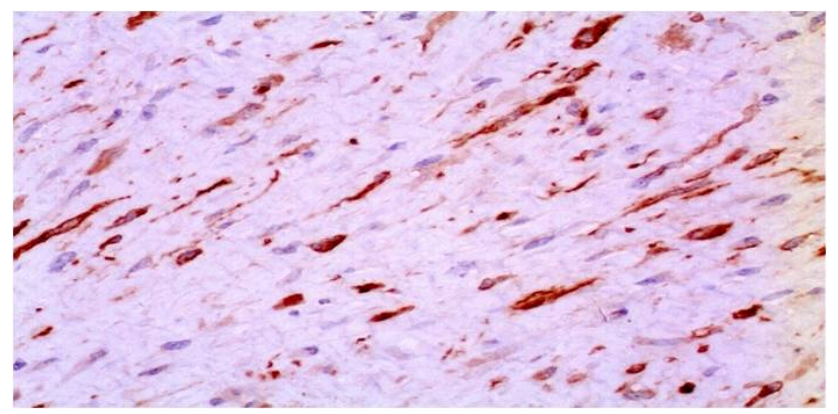

(c)

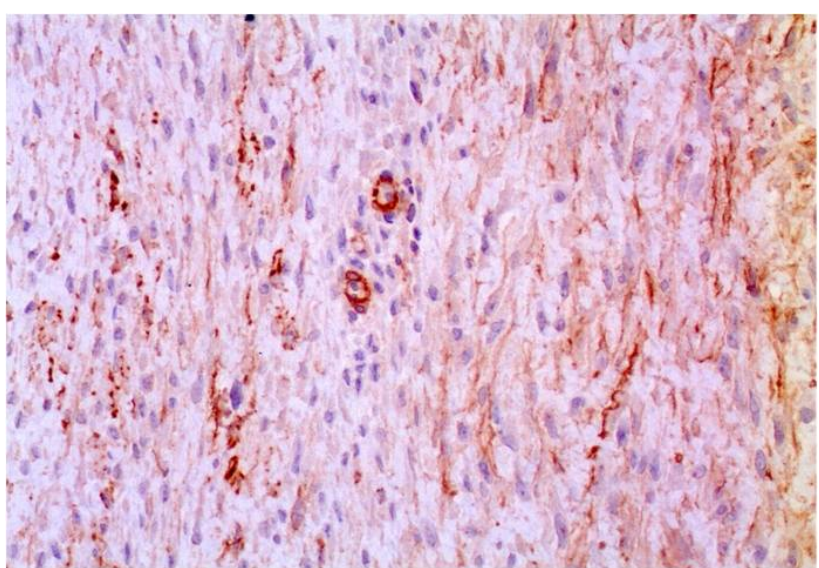

(b)

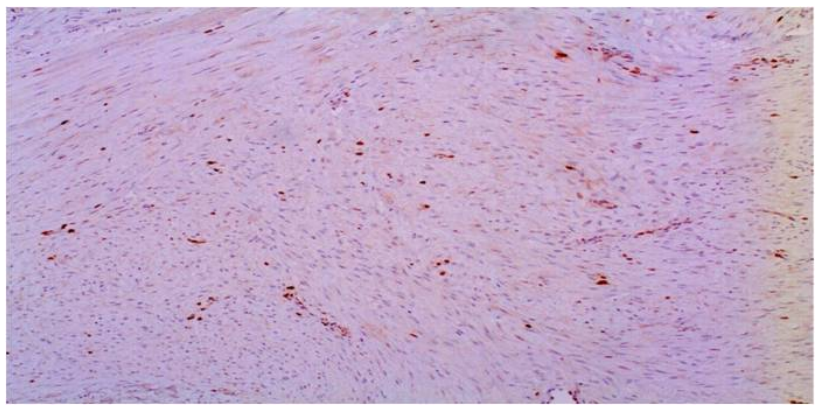

(d)

Figure 3. Histologically, the tumor consists of aggregates of elongated, spindle-shaped cells of uniform appearance, set in a collagenous stroma with prominent vascularity $(\mathrm{H} \& \mathrm{E}, \times 10)(\mathrm{a})$. The neoplastic cells demonstrate positive actin immunostaining (b) and variable smooth muscle desmin staining (c). The mitotic activity rate (ki-67) is low at less than $2 \%$ (d).

The reported incidence of sporadic DTs after surgery among adults $[2,7,19]$ varies between $14 \%$ and $33 \%$. Data for the pediatric population is limited. In a series of 63 children with DT in various sites, Faulkner et al. [20], noted that $11(17 \%)$ children had a history of local trauma prior to developing the tumor. Conversely, another study by Oudot et al. of 59 children with AF reported local trauma as a predisposing factor in only three (5\%) cases [21]. Honeyman et al. recently studied 93 children with DT [22], of whom 17 (18\%) were reported to have a history of preceding trauma. The scant reports of DTs emanating from a laparotomy scar [15,23-25] quote an estimated incidence of less than $0.05 \%$ (220 of the total DTs examined).

Warren [26] proposed four criteria to be considered in the development of a DT from a surgical trauma: 1) prior integrity of the tumor site, 2) an injury severe enough to initiate proliferation of the cells, 3) a reasonable latent period, and 4) a tumor compatible with the scar tissue and anatomic location of the injury. However, the exact mechanism of laparotomy scar-related DT is not clear. Faulkner et al. [20] suggested that the lesion pre-exists in an inactive state, and is possibly triggered by trauma due to an abnormal proliferation of connective tissue. Urist
[27] postulated the presence of a systemic or hereditary factor. The role of hormones, especially estrogen, is more evident in the case of DTs arising in a Cesarean section scar during pregnancy [28]. However, this theory does not appear to apply in the case of younger females. Whether genetic mutations, mostly reported in FAP patients, are implicated in the development of abdominal wall DTs is yet to be determined.

Although CT and especially MRI are useful in defining the anatomic margins of the tumor and its resectability, they are unable to distinguish DTs from other masses [29]. The definitive diagnosis is set by the histologic and immunohistochemical examination of the tumor [30]. Microscopically, the tumor is composed of abundant collagen which surrounds spindle-shaped cells of possible myofibroblast origin [10]. Immunohistochemical investigation reveals positivity for muscle cell markers such as actin and desmin [10], with low k-67 mitotic activity [3].

There is no optimal treatment for DTs as yet, owing to the lack of prospective randomized studies; large pediatric series are retrospective $[5,20,22,31,32]$. Where feasible, the current treatment of choice for DTs is complete surgical excision with negative surgical margins $[5$, 
$22,32]$; the risk of recurrence reaches $16 \%$ as compared to $67 \%$ with positive surgical margins [5,20,33-36]. However, in the case of an incomplete resection and potential risk of morbidity due to a second operation, adjuvant treatment with chemotherapy and/or noncytotoxic drugs or radiotherapy is recommended [5,37]. Notwithstanding, the role of chemotherapy is questionable. Buitendijk et al. [34] showed that a combination of chemotherapy with vincristine, actinomycin-D and cyclophosphamide could benefit cases of incomplete DT excisions. This is in line with a study by Meazza et al. [32] that reported a better outcome in patients with unresectable DTs followed by delayed surgery. The role of irradiation in the treatment of DTs in children has yet to be established. Meazza et al. [32] found that patients who received radiotherapy postoperatively for local control of the disease failed to show an improved outcome, and speculated that a different biology, a more aggressive nature of the lesion, and different responsiveness of children to radiotherapy might play a role. This is consistent with a study by Rao et al. [35] that reported $80 \%$ disease recurrence in children who received radiotherapy. In addition, complications of radiotherapy such as growth retardation, fractures, skin necrosis, cellulitis, secondary malignancies, infertility, and cardiotoxicity raise questions concerning its use [5,34]. The efficacy of non-cytotoxic agents such as non-steroid anti-inflammatory drugs [38], anti-estrogen drugs [38], imatinib mesylate [39], and hydroxyurea [40] among the pediatric population remains to be determined [5]. As concerns tumors that either do not progress or are asymptomatic, an initial, conservative "wait and see" policy has recently been proposed by Neyman et al. [5,22], since surgery could lead to severe morbidity.

\section{CONCLUSION}

Despite the relative rarity of DTs, the presence of a mass in an old laparotomy scar should raise suspicion of the lesion. An appropriate pre-operative work-up is essential, and surgery with negative margins is the treatment of choice. A close follow-up is recommended.

\section{REFERENCES}

[1] Wu, C., Amini-Nik, S., Nadesan, P., et al. (2010) Aggressive fibromatosis (desmoid tumor) is derived from mesenchymal progenitor cells. Cancer Research, 70, 76907698. http://dx.doi.org/10.1158/0008-5472.CAN-10-1656

[2] Reitamo, J.J., Häyry, P., Nykyri, E., et al. (1982) The desmoid tumor. I. Incidence, sex-, age- and anatomical distribution in the Finnish population. American Journal of Clinical Pathology, 77, 665-673.

[3] Bölke, E., Krasniqi, H., Lammering, G., et al. (2009) Chest wall and intrathoracic desmoid tumors: Surgical experience and review of the literature. European Journal of Medical Research, 14, 240-243.

http://dx.doi.org/10.1186/2047-783X-14-6-240

[4] Phillips, S.R., A’Hern, R. and Thomas, J.M. (2004) Aggressive fibromatosis of the abdominal wall, limbs and limb girdles. British Journal of Surgery, 91, 1624-1629. http://dx.doi.org/10.1002/bjs.4792

[5] Honeyman, J.N. and La Quaglia, M.P. (2012) Desmoids tumors in the pediatric population. Cancers, 4, 295-306. http://dx.doi.org/10.3390/cancers4010295

[6] Kulaylat, M.N., Karakousis, C.P., Keanay, C.M., et al. (1999) Desmoid tumor: A pleomorphic lesion. European Journal of Surgical Oncology, 25, 487-497. http://dx.doi.org/10.1053/ejso.1999.0684

[7] Häyry, P., Reitamo, J.J., Tötterman, S., et al. (1982) Desmoid tumor II. Analysis of factors possibly contributing to the etiology and growth behavior. American Journal of Clinical Pathology, 77, 674-680.

[8] Müller, J. (1838) Uber den feinern Bau und die Formen der Krankhften Creschwülste. G. Reimer, Berlin, 80.

[9] Macfarlane, J. (1832) In: Robertson, D., Ed., Clinical reports of the Surgical Practice of the Glasgow, Royal Infirmary, 63.

[10] Sakorafas, G.H., Nissotakis, C. and Peros, G. (2007) Abdominal desmoids tumors. Surgical Oncology, 16, 131142. http://dx.doi.org/10.1016/j.suronc.2007.07.009

[11] Reitamo, J.J., Scheinin, T.M. and Hayry, P. (1986) The desmoid syndrome: New aspects in the cause, pathogenesis and treatment of the desmoid tumour. American Journal of Surgery, 151, 230-237. http://dx.doi.org/10.1016/0002-9610(86)90076-0

[12] Friedl, W., Caspari, R., Sengteller, M., et al. (2001) Can APC mutation analysis contribute to therapeutic decisions in familial adenomatous polyposis? Gut, 48, 515-521. http://dx.doi.org/10.1136/gut.48.4.515

[13] Giarola, M., Wells, D., Mondini, P., et al. (1998) Mutations of adenomatous polyposis coli (APC) gene are uncommon in sporadic desmoid tumors. British Journal of Cancer, 78, 582-587. http://dx.doi.org/10.1038/bjc.1998.544

[14] Tejpar, S., Nollet, F., Li, C., et al. (1999) Predominance of beta-catenin mutations and beta-catenin dysrgeulation in sporadic aggressive fibromatosis (desmoids tumor). Oncogene, 18, 6615-6620. http://dx.doi.org/10.1038/sj.onc.1203041

[15] Economou, A., Pitta, X., Andreadis, A., et al. (2011) Desmoid tumor of the abdominal wall: A case report. Journal of Medical Case Reports, 5, 326. http://dx.doi.org/10.1186/1752-1947-5-326

[16] Casillas, J., Sais, G.J., Greve, J.L., et al. (1991) Imaging of intra- and extra abdominal desmoid tumors. Radiographics, 11, 959-968. http://dx.doi.org/10.1148/radiographics.11.6.1749859

[17] Brasfield, R.D. and Das Gupta, T.K. (1969) Desmoid tumors of the anterior abdominal wall. Surgery, 65, 241246.

[18] Weiss, S.W. and Goldblum, J.R. (2001) Fibromatoses. In: Enzinger and Weiss's Soft Tumors, Vol. 4, Mosb, St. 
Louis, 309-346.

[19] Stoeckle, E., Coindre, J.M., Longy, M., et al. (2009) A critical analysis of treatment in desmoids tumors: A review of a series of 106 cases. European Journal of Surgical Oncology, 35, 129-134. http://dx.doi.org/10.1016/j.ejso.2008.06.1495

[20] Faulkner, L.B., Hajdu, S.I., Kher, U., et al. (1995) Pediatric desmoids tumor $>$ retrospective analysis of 63 cases. Journal of Clinical Oncology, 13, 2813-2818.

[21] Oudot, C., Orbach, D., Minard-Colin, V., et al. (2012) Desmoid fibromatosis in pediatric patients: Management based on a retrospective analysis of 59 patients and a review of the literature. Sarcoma, 2012, 475202. http://dx.doi.org/10.1155/2012/475202

[22] Honeyman, J., Theilen, T.M., Knowles, M.E., et al. (2013) Desmoid fibromatosis in children and adolescents: A conservative approach to management. Journal of Pediatric Surgery, 48, 62-66. http://dx.doi.org/10.1016/j.jpedsurg.2012.10.017

[23] Berardi, R.S. and Canlas, M. (1973) Desmoid tumor and laparotomy scars. International Surgery, 58, 254-256.

[24] Overhaus, M., Decker, P., Fischer, H.P., et al. (2003) Desmoid tumor of the abdominal wall. World Journal of Surgical Oncology, 1, 11-15.

http://dx.doi.org/10.1186/1477-7819-1-11

[25] Wanjeri, J.K. and Opeya, C.J. (2011) A massive abdominal wall desmoids tumor occurring in a laparotomy scar: A case report. World Journal of Surgical Oncology, 9, 35. http://dx.doi.org/10.1186/1477-7819-9-35

[26] Warren, S. (1943) Minimal criteria required to prove causation of traumatic or occupational neoplasms. Annals of Surgery, 117, 585-595. http://dx.doi.org/10.1097/00000658-194304000-00008

[27] Urist, MR. (1957) Trauma and neoplasm: Report of case of desmoids tumor following simple fracture of the radius and ulna. American Journal of Surgery, 93, 682-688. http://dx.doi.org/10.1016/0002-9610(57)90531-7

[28] De Clan, F., Delay, E., Rudigoz, R.C., et al. (1999) Desmoid tumor arising in a Cesarean section scar during pregnancy: Monitoring and management. Gynecologic Oncology, 75, 145-148. http://dx.doi.org/10.1006/gyno.1999.5539

[29] Patrick, L.E., O’Shea, P., Simoneaux, S.F., et al. (1996) Fibromatoses of childhood: The spectrum of radiographic findings. American Journal of Roentgenology, 166, 163169. http://dx.doi.org/10.2214/ajr.166.1.8571869
[30] Sharma, A., Ngan, B.Y., Sãndor, G.K.B., et al. (2008) Pediatric aggressive fibromatosis of the head and neck: A 20-year retrospective review. Journal of Pediatric Surgery, 43, 1596-1604. http://dx.doi.org/10.1016/j.jpedsurg.2008.02.001

[31] Schmidt, D. and Harms, D. (1985) Fibromatosis of infancy and childhood-Histology and ultrastructure and clinicopathologic correlation. Kinderchir, 40, 40-46.

[32] Meazza, C., Bisogno, G., Gronchi, A., et al. (2010) Aggressive fibromatosis in children and adolescents: The Italian experience. Cancer, 116, 233-240.

[33] Ayala, A.G., Ro, J.Y., Goepfert, H., et al. (1986) Desmoid fibromatosis: A clinicopathologic study of 25 children. Seminars in Diagnostic Pathology, 3, 138-150.

[34] Buitendijk, S., van de Ven, C.P., Dumans, T.G., et al. (2005) Pediatric aggressive fibromatosis: A retrospective analysis of 13 cases and review of the literature. Cancer, 104, 1090-1099. http://dx.doi.org/10.1002/cncr.21275

[35] Rao, B.N., Horowitz, M.E., Rapala, M., et al. (1987) Challenges in the treatment of childhood fibromatosis. Archives of Surgery, 122, 1296-1298. http://dx.doi.org/10.1001/archsurg.1987.01400230082015

[36] Spiegel, D.A., Dormans, J.P., Meyer, J.S., et al. (1998) Aggressive fibromatosis from infancy to adolescence. Journal of Pediatric Orthopaedics, 19, 176-178.

[37] Merchant, T.E., Nguyen, D. and Walter, A.W. (2000) Long-term results with radiation therapy for pediatric desmoids tumors. International Journal of Radiation Oncology*Biology*Physics, 47, 1267-1271. http://dx.doi.org/10.1016/S0360-3016(00)00566-6

[38] Lackner, H., Urban, C., Kerbl, R., et al. (1997) Noncyotoxic drugs therapy in children with unresectable desmoid tumors. Cancer, 80, 334-340. http://dx.doi.org/10.1002/(SICI)1097-0142(19970715)80: 2<334::AID-CNCR22>3.0.CO;2-U

[39] Chugh, R., Wathen, J.K., Patel, S.R., et al. (2010) Efficacy of imatinib in aggressive fibromatosis: Results of a phase II multicenter Sarcoma Alliance for Research through Collaboration (SARC). Clinical Cancer Research, 16, 4884-4891. http://dx.doi.org/10.1158/1078-0432.CCR-10-1177

[40] Bisogno, G., Tagarelli, A., Stramare, R., et al. (2013) Hydroxyurea treatment can avoid the need for aggressive surgery in pediatric fibromatosis. Journal of Pediatric Hematology/Oncology, 35, e171-e173. http://dx.doi.org/10.1097/MPH.0b013e3182678d25 